\title{
A Two-Microphone Noise Reduction System for Cochlear Implant Users with Nearby Microphones-Part II: Performance Evaluation
}

\author{
Martin Kompis, ${ }^{1}$ Matthias Bertram, ${ }^{1,2}$ Pascal Senn, ${ }^{1}$ Joachim Müller, ${ }^{3}$ \\ Marco Pelizzone, ${ }^{4}$ and Rudolf Häusler ${ }^{1}$ \\ ${ }^{1}$ Department of ENT, Head and Neck Surgery Inselspital, University of Berne, 3010 Bern, Switzerland \\ ${ }^{2}$ Bernafon Inc., 3018 Bern, Switzerland \\ ${ }^{3}$ ENT clinic of the University of Würzburg, 97080 Würzburg, Germany \\ ${ }^{4}$ Clinique O.R.L., Hôpital Universitaire de Genève, 1211 Genève, Switzerland
}

Correspondence should be addressed to Martin Kompis, martin.kompis@insel.ch

Received 27 November 2007; Accepted 20 March 2008

Recommended by Chein-I Chang

\begin{abstract}
Users of cochlear implants (auditory aids, which stimulate the auditory nerve electrically at the inner ear) often suffer from poor speech understanding in noise. We evaluate a small (intermicrophone distance $7 \mathrm{~mm}$ ) and computationally inexpensive adaptive noise reduction system suitable for behind-the-ear cochlear implant speech processors. The system is evaluated in simulated and real, anechoic and reverberant environments. Results from simulations show improvements of 3.4 to $9.3 \mathrm{~dB}$ in signal to noise ratio for rooms with realistic reverberation and more than $18 \mathrm{~dB}$ under anechoic conditions. Speech understanding in noise is measured in 6 adult cochlear implant users in a reverberant room, showing average improvements of 7.9-9.6 dB, when compared to a single omnidirectional microphone or $1.3-5.6 \mathrm{~dB}$, when compared to a simple directional two-microphone device. Subjective evaluation in a cafeteria at lunchtime shows a preference of the cochlear implant users for the evaluated device in terms of speech understanding and sound quality.
\end{abstract}

Copyright (c) 2008 Martin Kompis et al. This is an open access article distributed under the Creative Commons Attribution License, which permits unrestricted use, distribution, and reproduction in any medium, provided the original work is properly cited.

\section{INTRODUCTION}

Unsatisfactory speech understanding in noise is a major complaint of users of cochlear implant systems $[1,2]$, even for users with acceptable levels of speech understanding in situations without background noise [3]. One method to alleviate this problem is the use of directional multimicrophone noise reduction systems, which reduce noise arriving from the sides or from the back of the cochlear implant user, while preserving signals arriving from the front.

In a companion paper [4], we presented a computationally inexpensive algorithm, which can be used with two nearby microphones mounted in a behind-the-ear speech processor. Supporting algorithms were developed and evaluated in simulated anechoic and reverberant environments $[4,5]$.

The aim of the study presented in this paper is to evaluate the performance of the proposed system [4] in simulated and real acoustic environments, and to perform physical tests as well as speech intelligibility tests with actual cochlear implant users.

This paper is organized as follows. Section 2 gives summary of the evaluated algorithm and of the hardware used. In Section 3, the proposed algorithm is evaluated in simulated anechoic and reverberant environments. Section 4 describes physical measurements performed in a real anechoic chamber and in a reverberant room. In Section 5, we report on speech intelligibility tests with 6 adult cochlear implant users in a well-defined experimental setting. Section 6 summarizes the subjective assessment of 6 users in a noisy cafeteria.

\section{BEAMFORMING ALGORITHM AND EXPERIMENTAL DEVICE}

Figure 1 shows a schematic diagram of the algorithm under evaluation. It was implemented in a real-time prototype 


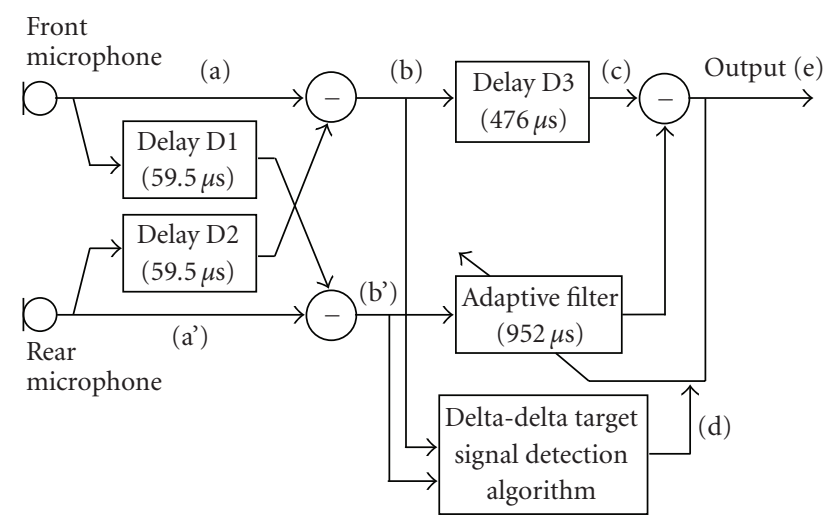

FIGURE 1: Block diagram of the beamforming algorithm implemented in the experimental real-time device. The two microphones are mounted in a behind-the-ear housing (intermicrophone distance $7 \mathrm{~mm}$ ).

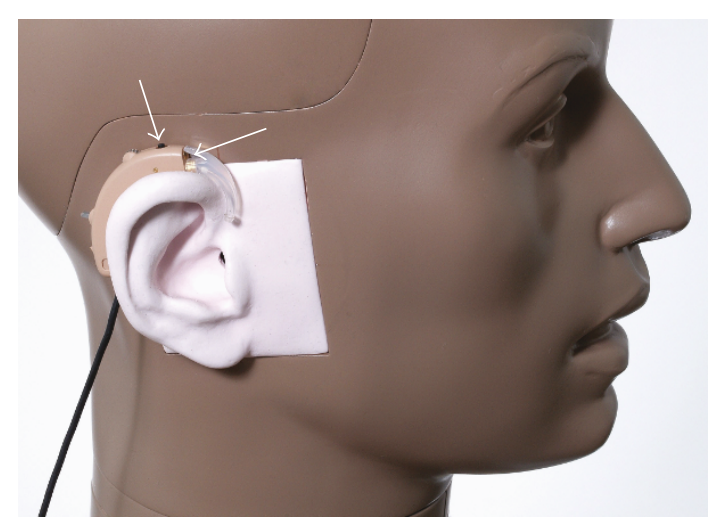

Figure 2: Behind-the-ear housing containing the two-omnidirectional microphones (arrows) mounted on KEMAR manikin.

device. Only a short summary is given here, a more detailed description can be found elsewhere [4].

The algorithm uses two nearby microphones, the output signals of which are combined to form two simple fixed directional units, one pointing forward (signal (b)), and one to the back (signal (b')). Signal (b) will contain predominantly signals from sources lying in front of the listener, signal ( $\left.b^{\prime}\right)$ predominantly noise. An adaptive filter then transforms the noise signal (b') into an estimate of the remaining noise on the delayed target signal (b). The difference between the noisy signal and this estimate is the output signal (c). A normalized LMS-algorithm $[6,7]$ is used for filter adaptation, resulting in a theoretical adaptation time constant of 2.4 milliseconds. Delay D3 is half of the length of the adaptive filter and intended to optimize its performance.

The delta-delta target signal detection scheme [4] continuously estimates the signal-to-noise ratio (SNR) at the input and interrupts filter adaptation during time segments with high SNRs, thus avoiding cancellation of the target signal and defining the opening angle of the device. The two omnidirectional microphones are mounted in a behind-theear (BTE) hearing aid housing, their acoustic ports separated by a distance of $7 \mathrm{~mm}$ (Figure 2). The algorithm itself is implemented on a portable digital signal processing (DSP) system built around a Motorola DSP56F826 processor. The experimental device can be used in 4 different modes. In mode (i), the output of the device is the output signal (e) of the adaptive beamformer using the algorithm and parameters above, in mode (ii), the signal of one of the omnidrectional microphones (Figure 1, signal (a)) is routed directly to the output, in mode (iii), the output of the directional fixed unit pointing to the front (Figure 1, signal (b)) is routed the output of the device, and in mode (iv), the coefficients of the adaptive filter are frozen until mode (i) is restored.

\section{EVALUATION OF SIMULATED ENVIRONMENT EXPERIMENTS}

The basic algorithm shown in Figure 1 was evaluated in two different simulated acoustic environments. While the amount of noise reduction can be predicted for similar devices with microphones placed above both ears of the user $[8,9]$ to date there is no such theoretical framework for the device in Figure 1.

The room simulation procedure used [5] is based on an image method and simulates impulse responses between acoustic sources and microphones in shoeboxshaped rooms, taking the head shadow of the listener into account. Heads are modeled as rigid spheres with a diameter

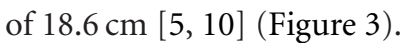

Two acoustic environments were simulated and used for this evaluation: one anechoic environment and one reverberant room. For the reverberant room, a reverberation time (i.e., time for the reverberant signal to decay by $60 \mathrm{~dB}$ ) of 0.4 seconds and a volume of $34 \mathrm{~m}^{3}$ were chosen, as these were the average values found from a series of 18 different rooms in our own environment [10]. Note, however, that these values may differ, for example, in a different cultural context.

Figure 3 shows a schematic drawing of the simulated rooms including a simple model for the head of the listener. 4-omnidirectional sound sources were placed at a distance 


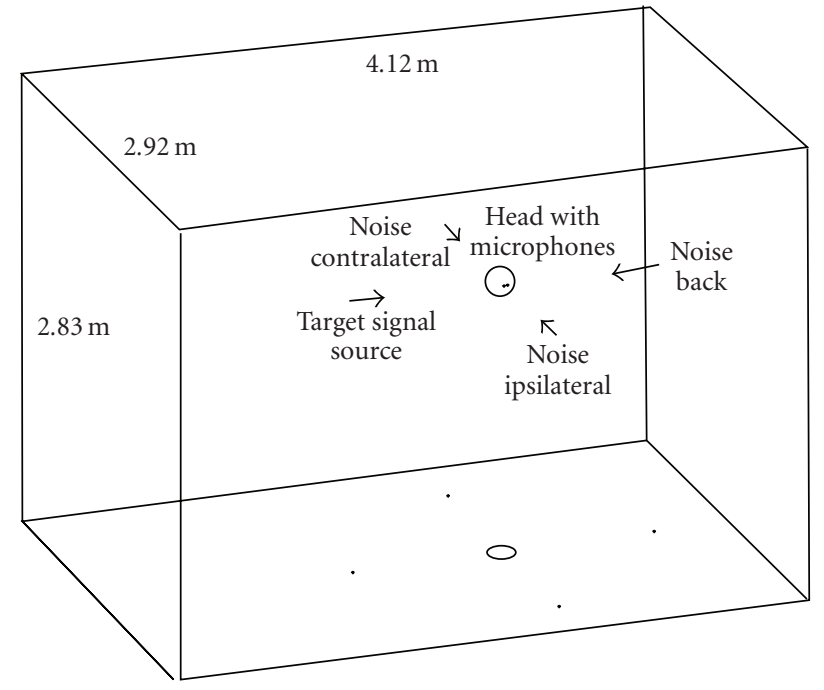

FIGURE 3: Schematic drawing of the simulated room including the head of the listener, modeled as a rigid sphere, and 4 sound sources at a distance of $1 \mathrm{~m}$ from the center of the head of the listener.

of $1 \mathrm{~m}$ from the center of the head of the listener. For the simulations, 3 different segments of 2 seconds duration white noise (sampling rate $10,000 \mathrm{~s}^{-1}$ ) were created. The first noise signal was filtered with the two different simulated impulse responses: one between the noise source and the front microphone on the surface of the model head, and one between the noise source and the rear microphone. These signals were then processed by the algorithm depicted in Figure 1, but the length of the adaptive filter was varied systematically, and an ideal target signal detection algorithm performance was mimicked by allowing the filter adapt only in the absence of the target signal. Delay D3 was set to one half of the length of the adaptive filter in all experiments. A normalized LMS-adaptation algorithm with an adaptation time constant of $10 \%$ of the value, which is expected to lead to instability was used [7]. The coefficients of the adapted filter were saved at the end of this step. Then, the second noise signal was processed in the same way, except that the filter coefficients remained frozen in the adapted state from the first run. Then, the third noise signal was filtered by the impulse responses between the target source infront of the listener, as depicted in Figure 3, and the two microphones and processed by the beamforming algorithm with its adaptive filter coefficients still frozen in the adapted state. The output signals (e) of these last two runs were used to estimate the maximum obtainable noise reduction. In this way, an idealized situation with the filter being adapted in the absence of the target signal was obtained.

The above procedure was repeated for all combinations of (i) 3 directions of incidence of the noise signal (ipsilateral to the microphones, contralateral to the microphones and from the back, Figure 2), (ii) two rooms (anechoic and reverberant), and (iii) 3 lengths of the adaptive filter (1 millisecond, 10 milliseconds, and 50 milliseconds).

Figure 4 shows a summary of the results. In addition to the results of the adaptive algorithm, the noise reduction

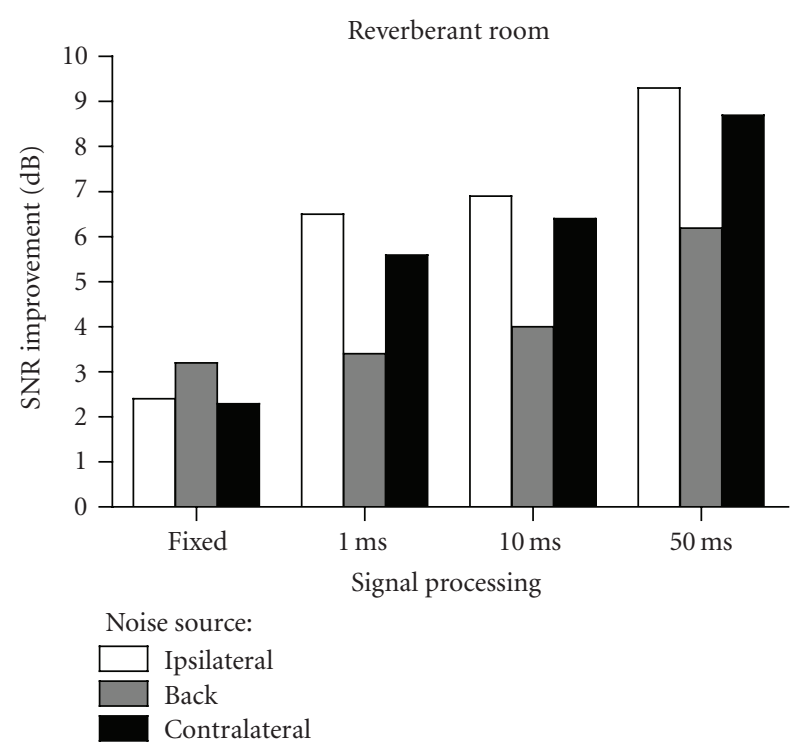

(a)

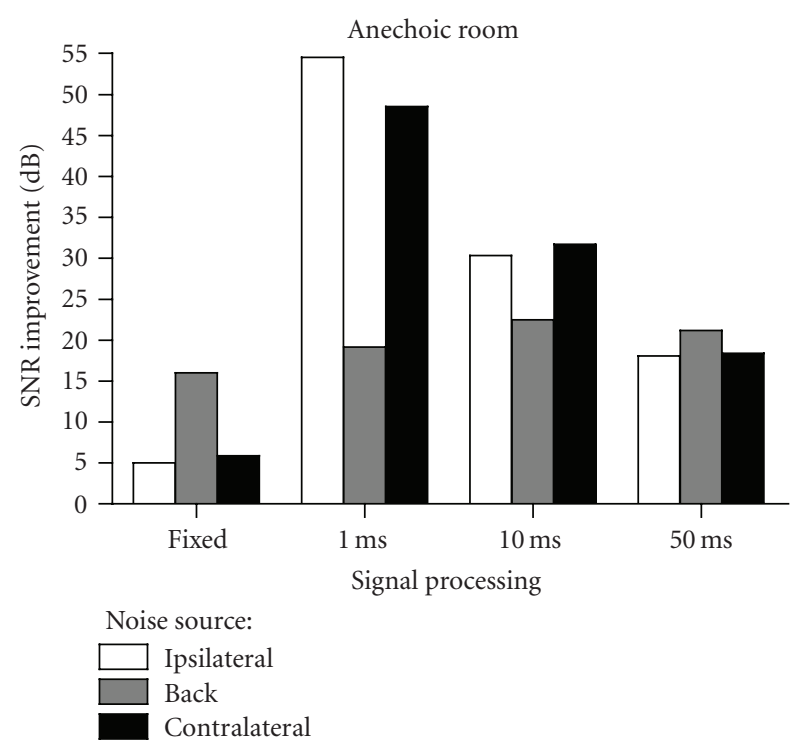

(b)

FIGURE 4: SNR improvements of the two-microphone noise reduction system. Top panel: results from simulations in a reverberant room with a reverberation time of 0.4 seconds. Bottom panel: simulations in an anechoic room. Noise reductions are shown as a function of the direction of incidence of the noise and the signal processing used. Label "Fixed": simple fixed directional system, corresponding to signal (b) in Figure 1. Labels "1 millisecond", "10 milliseconds", and "50 milliseconds": output of an adaptive system corresponding to signal (e) in Figure 1, using the given length for the adaptive filter.

of a simple fixed two-microphone directional system (signal (b) in Figure 1) was included. In this way, comparisons to other published algorithms, which also use the SNR of an omnidirectional microphone and/or a simple directional microphone as a baseline $[10,11]$, are facilitated. All improvements are standardized to the signal-to-noise ratio at the front microphone without any signal processing. 


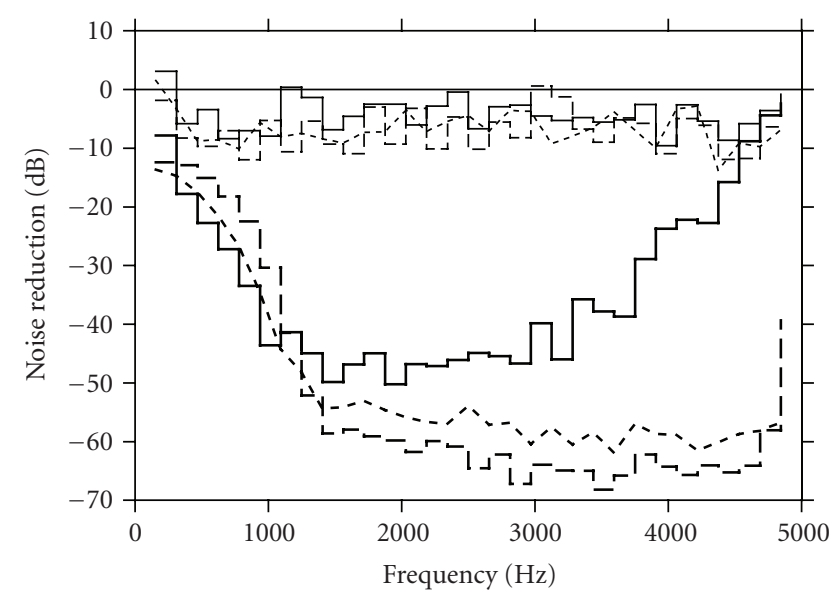

$\begin{array}{ll}\text { Reverberant room: } & \text { Anechoic room: } \\ \text {--- Noise ipsilateral } & --- \text { Noise ipsilateral } \\ \text { - Noise back } & - \text { Noise back } \\ \text {---- Noise contralateral } & \text {---- Noise contralateral }\end{array}$

FIGURE 5: Noise reduction of the adaptive two-microphone system (length of the adaptive filter 10 milliseconds) in 32 frequency bins in simulated reverberant and anechoic environments.

In the reverberant room, noise reductions range between 3.4 to $9.3 \mathrm{~dB}$ for the adaptive beamformers, with the improvements increasing with the filter length. For each direction of incidence and for any of the different filter lengths, the adaptive system outperforms the fixed twomicrophone system. The difference is the largest, if the noise source is positioned ipsilateral to the microphones, and the smallest if the noise arrives from the back.

In the anechoic environment, improvements are larger and range from 18 to $54 \mathrm{~dB}$. Adaptation time, that is, the time required to reach a stable amount of noise reduction in a given environment, increases with the adaptation time constant and with the amount of noise reduction that can be reached in the adapted state. As longer filters result in proportionally longer adaptation time constants, the adaptation time of 2 seconds allowed in these offline experiments becomes too short to reach a steady state for the two long filters, resulting in a lower (but still impressive) noise suppression.

The adaptive filter may, in principle, change the frequency response of the system and might, for example, suppress predominantly signals in frequency regions, which do not contribute substantially to speech intelligibility. To investigate this effect, the output of the simulations was analyzed in the frequency domain. Figure 5 shows the results for adaptive filters of 10 milliseconds in length. It can be seen that the frequency response of the noise reduction is reasonably flat for the situations in the reverberant room, but poorer at the lower frequencies in the anechoic environment. For noise arriving from the back, signal portions toward the higher frequencies above approximately $3500 \mathrm{~Hz}$ are also affected. Although comparable frequency responses have been reported for other systems using adaptive finite impulse filters [10-12], to our knowledge, the reason for this behavior is not yet fully understood. Figure 5 is typical also for other filter lengths, in that the response is flat in the reverberant environment and greatest in the middle-to-high frequencies in the anechoic room.

These simulations give a first idea on the performance of the proposed device under idealized conditions. However, tests in real acoustic environments are needed to assess, whether similarly favorable results can be achieved under more realistic conditions.

\section{PHYSICAL EVALUATION}

The device was evaluated physically using two different real acoustic environments: an anechoic chamber and a moderately reverberant room with a nearly frequency independent reverberation time of 0.37 second $(250 \mathrm{~Hz}-4000 \mathrm{~Hz})$, and a volume of $42 \mathrm{~m}^{3}$. This room was chosen, as it was conveniently available for measurements and its acoustic properties were reasonably close to that of an imaginary average room found in an earlier study [10], and to the simulated reverberant room used in the companion paper [4].

For the physical evaluation in both rooms, the BTE unit holding both microphones was placed behind the ear of a knowles electronic manikin for acoustic research (KEMAR, Figure 2). A loudspeaker emitting white noise was placed $1 \mathrm{~m}$ away from the center of the head of the manikin and moved around the manikin in steps of $10^{\circ}$. The output of the experimental device described in Section 2 was measured in three different conditions: output of the omnidirectional (front) microphone, output of the fixed beamformer pointing to the front (signal (b) in Figure 1), and the output (c) of the adaptive beamformer. For the last measurement, the filter was first adapted during 1 second, and then the filer coefficients were frozen. Using these filter coefficients, the output of the beamformer was compared to white noise arriving at an azimuth of $0^{\circ}$ (target signal) and at the current position of the noise source. All SNR improvements were spectrally weighted using intelligibilityweighted gains [13] for better correspondence to actual improvements in speech understanding.

Figure 6 shows the results of the measurements. On the left-hand side of Figure 6, the results of the measurements in the anechoic room are shown. On the right-hand side, results from the reverberant room can be seen. The top row shows the polar plot of the omnidirectional microphone mounted to the left side of the head $\left(90^{\circ}\right)$ of the KEMAR, the middle row the corresponding plot for the fixed directional processing, and the bottom row the plot for the proposed adaptive beamformer.

Already for the omnidirectional microphone, there is some limited directionality mainly in the direction of the placement of the BTE-unit at the $\operatorname{KEMAR}\left(90^{\circ}\right)$. For the fixed directional processing, the directional lobe moves partly toward the front $\left(0^{\circ}\right)$, and signals arriving from the sides and the back $\left(90^{\circ}\right.$ to $\left.270^{\circ}\right)$ are attenuated less in the reverberant than in the anechoic environment. The most pronounced directionality can be seen in the output of the adaptive beamformer (bottom row of Figure 6) with noise 


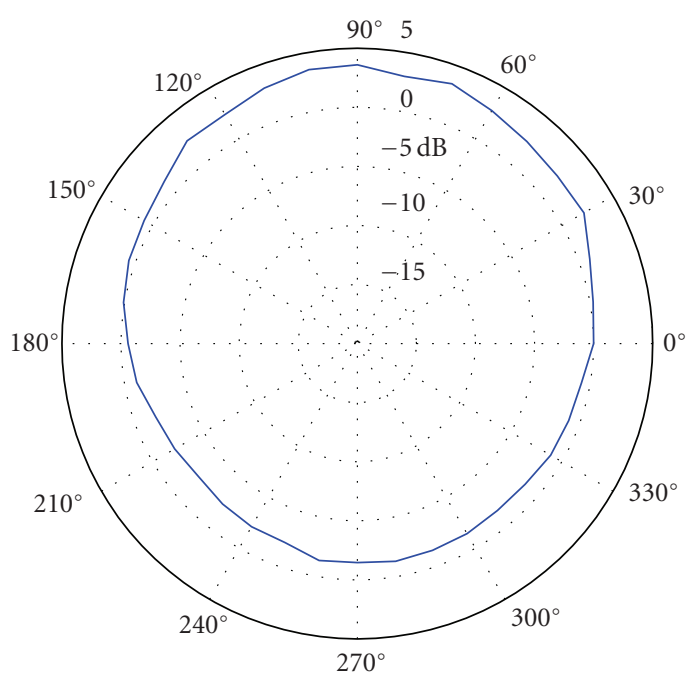

(a)

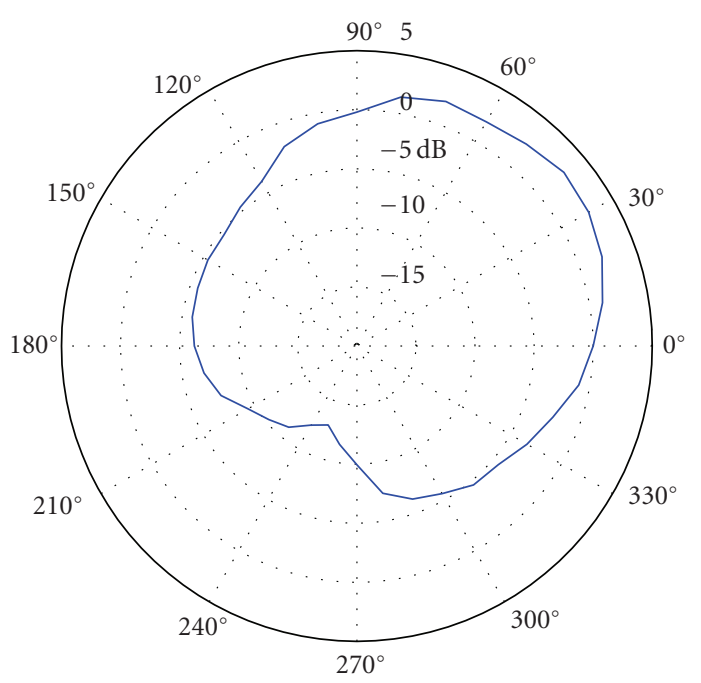

(c)

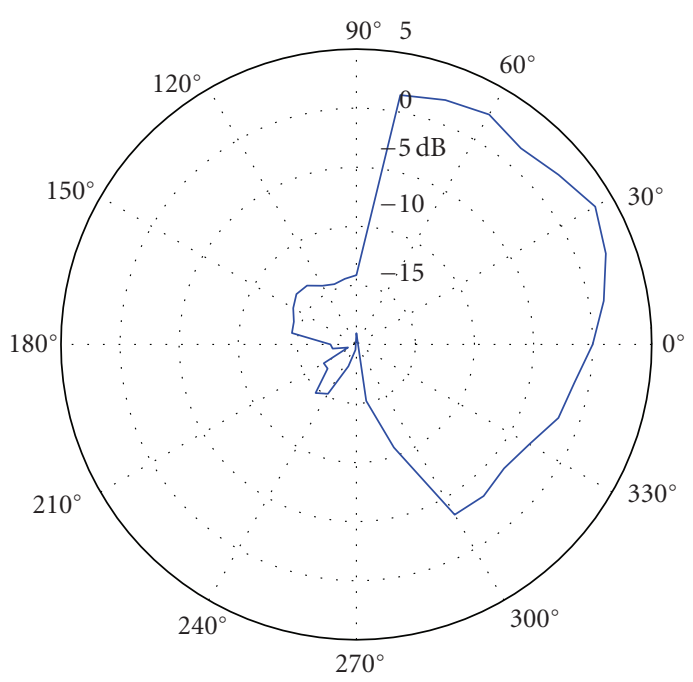

(e)

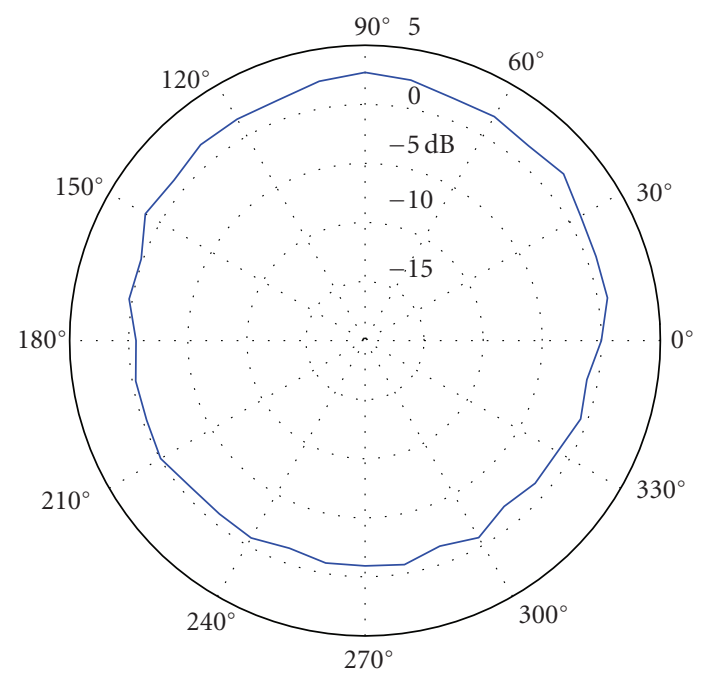

(b)

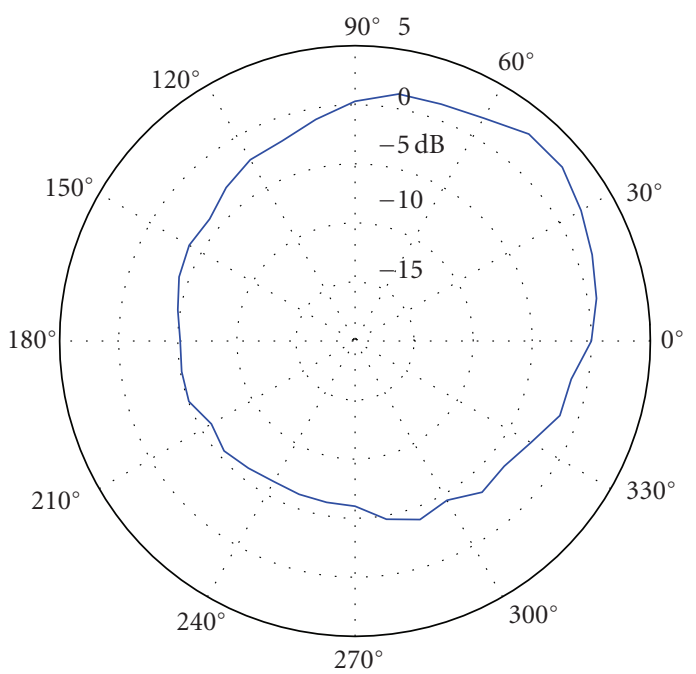

(d)

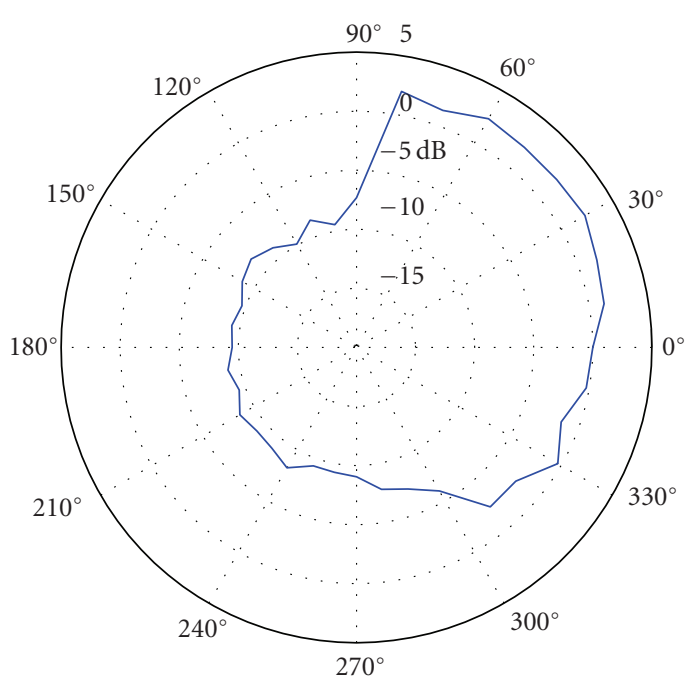

(f)

FIgure 6: Polar plots of device performance in three different modes. The behind-the-ear unit with the microphones is mounted at the left side $\left(90^{\circ}\right)$ of a KEMAR manikin, facing $0^{\circ}$. Top row: one-omnidirectional microphone. Middle row: fixed directional processing. Bottom row: adaptive beamformer. Left column: results from measurements in an anechoic chamber. Right column: results in reverberant room. 


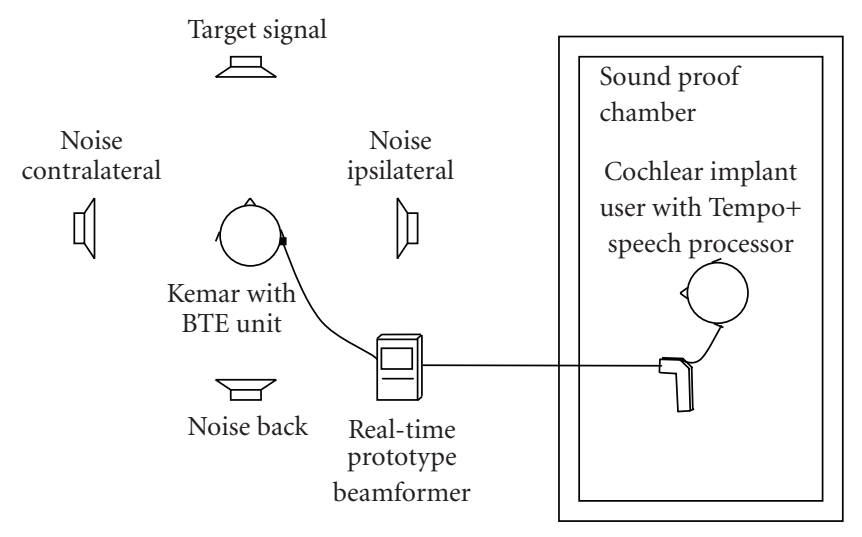

FIgURE 7: Experimental setting for speech intelligibility tests in noise with cochlear implant users.

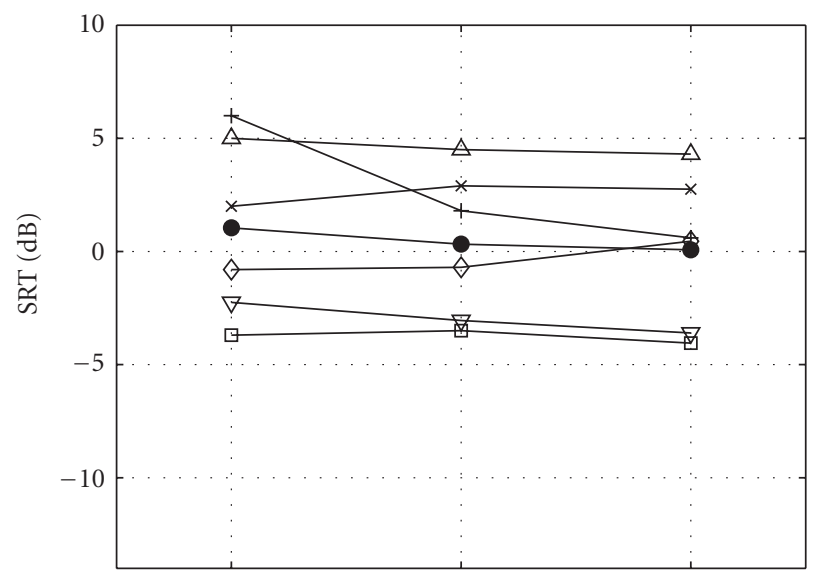

Omnidirectional mic Fixed directional mic Beamformer

$$
\begin{array}{ll}
* \mathrm{~S} 1 & \triangle \mathrm{S} 5 \\
\neg \mathrm{S} 2 & +\mathrm{S} 6 \\
\square \mathrm{S} 3 & -\mathrm{-} \text { Mean } \\
\nabla \mathrm{S} 4 &
\end{array}
$$

FIGURE 8: Speech reception thresholds (SRTs) of six cochlear implant users for target and noise signals arriving from the front.

attenuations around 7.5 to almost $10 \mathrm{~dB}$ under reverberant conditions $\left(90^{\circ}\right.$ to $\left.270^{\circ}\right)$ and for some directions over $15 \mathrm{~dB}$ in the anechoic chamber. Over all, the beamforming part of the algorithm results in a substantial gain in SNR already in the reverberant room, and even more in the anechoic chamber.

\section{SPEECH INTELLIGIBILITY TESTS IN NOISE WITH COCHLEAR IMPLANT USERS}

Speech intelligibility tests are an important part of the evaluation, as not all—possibly detrimental-effects of our algorithm can be assessed using physical measures alone.

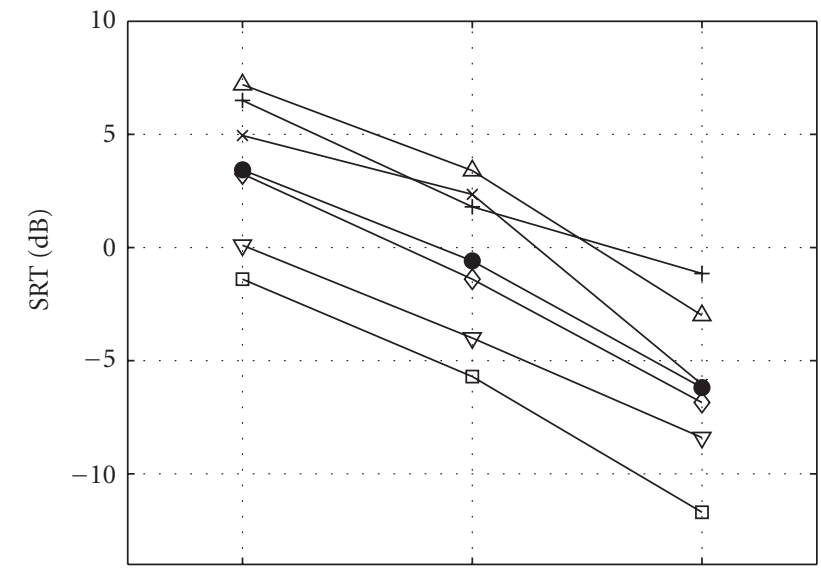

Omnidirectional mic Fixed directional mic Beamformer

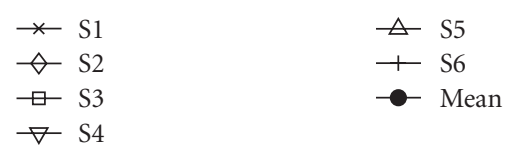

FIGURE 9: Speech reception thresholds (SRTs) of six cochlear implant users for noise arriving from the side ipsilateral to the behind-the-ear microphone unit.

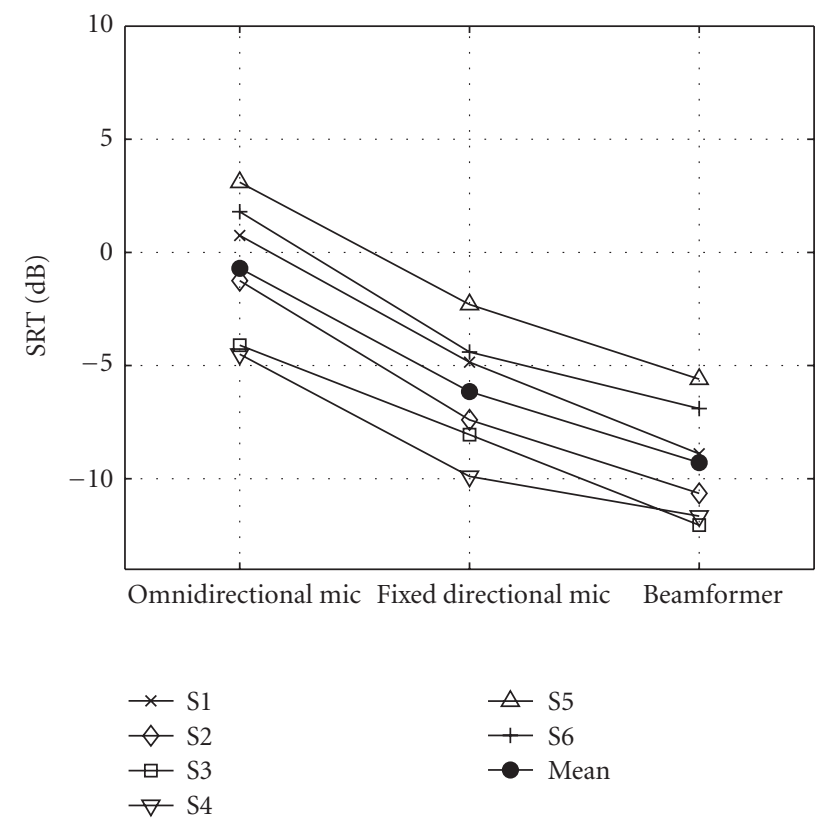

FIGURE 10: Speech reception thresholds (SRT) of six cochlear implant users for noise arriving from the side contralateral to the behind-the-ear microphone unit.

\subsection{Subjects}

6 adult cochlear implant users ( 2 women, 4 men, ages $37-$ 65, mean 52 years) participated in the study. 5 had a Medel Combi+ device implanted in their left ear, one person had the same type of implant in his right ear. All used Medel 


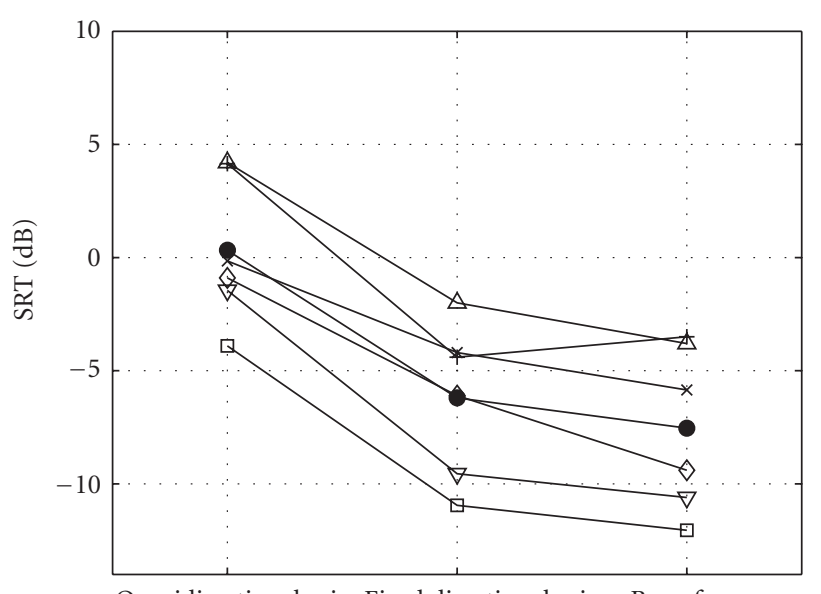

Omnidirectional mic Fixed directional mic Beamformer

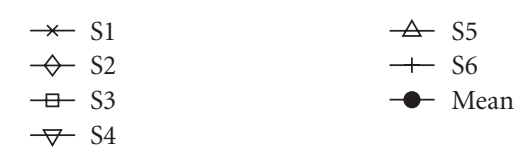

FIgURE 11: Speech reception thresholds (SRT) of six cochlear implant users for noise arriving from the back, and the target signal arriving from the front.

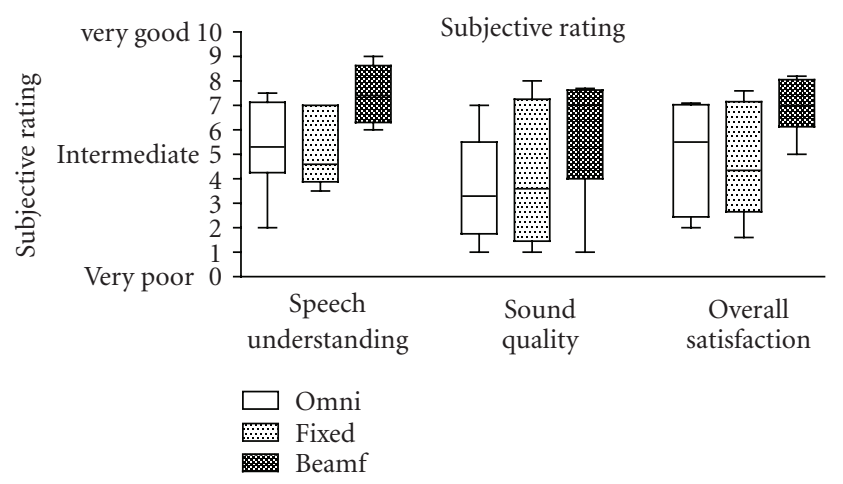

FIGURE 12: Subjective assessment of three signal processing listening conditions by the cochlear implant users in a cafeteria at lunch time. Box plots denote minima, maxima, and quartiles of the subjective ratings on a visual analogue scale.

Tempo+ speech head level speech processors [14] for at least 1 year.

\subsection{Experimental protocol}

The experimental protocol was approved by the local ethical committee and informed consent was obtained from all subjects prior to the experiments. Figure 7 shows a schematic drawing of the experimental setting. All experiments were performed in the moderately reveberant room described above (reverberation time 0.37 second). The BTE unit containing the two microphones was mounted behind the ear of a KEMAR (Figure 2). 4 loudspeakers were placed around the KEMAR at a distance of $1 \mathrm{~m}$; one in front, always emitting the speech signal, one on each side and one in the back. The microphone signals were processed by the portable experimental beamformer device and routed into a modified Medel Tempo+ speech processor, the microphone of which had been removed. Subjects were sitting in a separate sound proof chamber. This was not strictly necessary as the patients are profoundly deaf, but ensures compatibility with test with normally hearing subjects.

Speech intelligibility was tested using the German Olderburger sentence test [15]. From this test material, 30 grammatically correct sentences consisting of 5 words each was presented to the subject through the front loudspeaker. Competing speech babble noise was presented either by the front, the right, the left, or the rear loudspeaker. The SNR of each presentation was varied adaptively according to the instructions of the Olderburger sentence test in order to estimate the SNR level required for 50\% understanding of the words in each sentence.

3 different conditions were tested: (1) no signal processing (signal of the omnidirectional front microphone routed through the experimental system), (2) fixed directional microphone, that is, signal (b) in Figure 1 routed to the speech processor of the subject, and (3) output of the adaptive beamformer (signal (e) in Figure 1). The order of the tests was varied systematically to minimize effects of training or fatigue. Results were analyzed statistically using a linear mixed model [16] and were Bonferroni corrected for the effect of multiple testing.

\subsection{Results of the speech intelligibility tests}

Figure 8 shows the speech reception thresholds (SRTs) if both the signal and the noise arrive from the front of the listener. As can be expected, there is no advantage of either directional processing, when the two sources cannot be separated spatially. This part of the experiment was used to confirm that no detrimental effects are introduced by the fixed or adaptive signal processing in the experimental DSP device.

Figure 9 shows speech reception thresholds (SRTs) if the noise arrives from the side ipsilateral to the BTE microphone unit at the KEMAR. SRTs are now improved by the adaptive beamformer, on average, by $9.6 \mathrm{~dB}$, when compared to the omnidirectional microphone, and by $5.6 \mathrm{~dB}$, when compared to the fixed directional microphone unit. The differences are statistically significant $(P<.03)$.

Figure 10 shows the SRTs for speech babble noise emitted by loudspeaker contralateral to the side of the KEMAR wearing the BTE microphone unit. The average improvement by the adaptive beamformer is $8.6 \mathrm{~dB}$ compared to the omnidirectional microphone and $3.2 \mathrm{~dB}$ compared to the fixed directional unit. Again, all differences are statistically significant $(P<.03)$.

Figure 11 finally shows the SRT improvements for noise arriving from the back of the KEMAR. The improvements are somewhat smaller, $7.8 \mathrm{~dB}$, when compared to the omnidirectional microphone, and $1.3 \mathrm{~dB}$, when compared to the fixed directional microphone unit. 


\section{SUBJECTIVE EVALUATION}

Subjective evaluations by the users are important, as they correlate with the benefit of the system, as perceived by the user. However, systematic subjective evaluations are complex, time consuming, and may give equivocal results even for a substantial measurable benefit and if validated questionnaires are used $[11,17]$. Although such a systematic study is beyond the scope of the presented research project, we were interested to learn how the effect of an adaptive beamformer is perceived in an acoustically complex but realistic situation.

Each of the 6 cochlear implant user spent 20 minutes in a busy hospital cafeteria at lunch time. They were equipped with the experimental beamforming device and were allowed to switch between the omnidirectional microphone, the fixed directional microphone unit, and the adaptive beamformer. They were given 9 different analog visual scales labelled equidistantly from 0 (very poor) to 10 (very good) and asked to rate speech understanding of another person seated at the same table, sound quality, and overall satisfaction with each of the three settings.

Figure 12 shows a summary of the results of the survey. All three aspects are rated better by all 6 subjects for the adaptive beamformer than for the other two processing conditions, with the single exception of a user, who rated the sound quality in the cafeteria as very poor for all 3 processing options. All differences between the adaptive beamformer and the other two conditions are statistically significant $(P<$ .05, Wilcoxon matched pairs test). The differences between the omnidirectional microphone and the fixed directional microphone units are not significant.

\section{DISCUSSION AND SUMMARY}

A directional, adaptive two-microphone noise reduction system was evaluated. Experiments in simulated acoustic environments predict a very large gain in anechoic environments and gains of 3.4 to $9.3 \mathrm{~dB}$ in rooms with realistic amounts of reverberation, compared to a simple directional nonadaptive two-microphone system.

The performance of a prototype device was evaluated in an anechoic chamber and in a reverberant room. With an adaptive filter of less than 1 millisecond, the evaluated device is computationally relatively inexpensive. With a distance of only $7 \mathrm{~mm}$ between the microphone ports, a physically small implementation in a BTE speech processor for cochlear implant system seems possible. The benefit in terms of improved speech intelligibility in noise in a real environment with realistic amounts of reverberation is substantial ( 7.9 to $9.6 \mathrm{~dB}$ ), when compared to a single omnidirectional microphone. Today, the speech processors for cochlear implant systems of several manufacturers are still using single omnidirectional microphones [17, 18]. Compared to the more complex beamforming algorithm of one cochlear implant manufacturer [11], the computational load is smaller, as is the physical size of the device $(7 \mathrm{~mm}$ intermicrophone distance instead of $19 \mathrm{~mm}$ ). However, the noise reduction is probably also somewhat lower [11].
There is a good agreement between the physical measurements and the SRT improvement in the speech intelligibility tests, indicating that the potential found in the physical measurements can be largely used by the cochlear implant users to improve speech understanding.

Speech intelligibility tests were performed with a single stationary noise source only. However, the test in the cafeteria with many different moving noise sources has shown that the evaluated device remains beneficial for the patients even in much more complex acoustic conditions. A subjective evaluation study has shown that this benefit is also perceived subjectively by the cochlear implant users.

In conclusion, the evaluated beamforming device seems suitable for the application in behind-the-ear speech processors for cochlear implants.

\section{ACKNOWLEDGMENTS}

This work was supported by the Swiss National Science Foundation, Grant no. 3238-056325/2 and by a Grant from the Medel Clinical Research Fund.

\section{REFERENCES}

[1] J. Müller, F. Schon, and J. Helms, "Speech understanding in quiet and noise in bilateral users of the MED-EL COMBI 40/40+ cochlear implant system," Ear and Hearing, vol. 23, no. 3, pp. 198-206, 2002.

[2] M. Kompis, M. Bettler, M. Vischer, P. Senn, and R. Häusler, "Bilateral cochlear implantation and directional multimicrophone systems," in Cochlear Implants, R. Miyamoto, Ed., Elsevier International Congress Series, pp. 447-450, Elsevier, Amsterdam, The Netherlands, 2004.

[3] M. Kompis, M. Jenk, M. W. Vischer, E. Seifert, and R. Häusler, "Intra- and intersubject comparison of cochlear implant systems using the Esprit and the Tempo+ behind-the-ear speech processor," International Journal of Audiology, vol. 41, no. 8, pp. 555-562, 2002.

[4] M. Kompis, B. Bertram, J. François, and M. Pelizzone, "A twomicrophone noise reduction system for cochlear implant users with nearby microphones-Part I: signal processing algorithm design and development," to appear in EURASIP Journal on Advances in Signal Processing.

[5] M. Kompis and N. Dillier, "Simulating transfer functions in a reverberant room including source directivity and headshadow effects," The Journal of the Acoustical Society of America, vol. 93, no. 5, pp. 2779-2787, 1993.

[6] B. Widrow, J. R. Glover Jr., J. M. McCool, et al., "Adaptive noise cancelling: principles and applications," Proceedings of the IEEE, vol. 63, no. 12, pp. 1692-1716, 1975.

[7] S. Haykin, Adaptive Filter Theory, Prentice-Hall, Upper Saddle River, NJ, USA, 4th edition, 2001.

[8] M. Kompis and N. Dillier, "Performance of an adaptive beamforming noise reduction scheme for hearing aid applications-I: prediction of the signal-to-noise-ratio improvement," The Journal of the Acoustical Society of America, vol. 109, no. 3, pp. 1123-1133, 2001.

[9] M. Kompis, "A measuring medical pocket calculator," Medical Equipment Insights, vol. 1, pp. 3-7, 2008.

[10] M. Kompis and N. Dillier, "Performance of an adaptive beamforming noise reduction scheme for hearing aid applications-II: experimental verification of the predictions," 
The Journal of the Acoustical Society of America, vol. 109, no. 3, pp. 1134-1143, 2001.

[11] A. Spriet, L. van Deun, K. Eftaxiadis, et al., "Speech understanding in background noise with the two-microphone adaptive beamformer BEAM $^{\mathrm{TM}}$ in the nucleus Freedom ${ }^{\mathrm{TM}}$ cochlear implant system," Ear and Hearing, vol. 28, no. 1, pp. 62-72, 2007.

[12] P. Müller and M. Kompis, "Evaluation of a noise reduction system for the assessment of click-evoked otoacoustic emissions," The Journal of the Acoustical Society of America, vol. 112, no. 1, pp. 164-171, 2002.

[13] J. E. Greenberg, P. M. Peterson, and P. M. Zurek, "Intelligibility-weighted measures of speech-to-interference ratio and speech system performance," The Journal of the Acoustical Society of America, vol. 94, no. 5, pp. 3009-3010, 1993.

[14] J. Helms, J. Müller, F. Schön, et al., "Comparison of the TEMPO+ ear-level speech processor and the CIS PRO+ bodyworn processor in adult MED-EL cochlear implant users," Journal for Oto-Rhino-Laryngology and Its Related Specialties, vol. 63, no. 1, pp. 31-40, 2001.

[15] K. Wagener, V. Kühnel, and B. Kollmeier, "Entwicklung und evaluation eines satztests für die deutsche sprache I: design des oldenburger satztests," Zeitschrift für Audiologie, vol. 38, no. 1, pp. 4-15, 1999.

[16] H. Brown and R. Prescott, Applied Mixed Models in Medicine, John Wiley \& Sons, West Sussex, UK, 1999.

[17] Medel Inc., "OPUS 2 Speech Processor," 2006, http://www. medel.com/.

[18] Advanced Bionics Inc., "Guide to the Auria Harmony Speech Processor,” 2007, http://www.bionicear-europe.com/. 